\title{
Riesgo de déficit atencional/hiperactividad en escolares Aymara, Rapa-Nui y de Santiago de Chile. Posible contribución de polimorfismos genéticos del sistema dopaminérgico
}

\author{
LIZA PAZ LAGOS ${ }^{1, \mathrm{a}}$, CLAUDIO SILVA ${ }^{2}$, PAULA ROTHHAMMER ${ }^{4}$, \\ XIMENA CARRASCO ${ }^{5}$, ELENA LLOP ${ }^{3}$, \\ FRANCISCO ABOITIZ ${ }^{4}$, FRANCISCO ROTHHAMMER ${ }^{1,3}$
}

Instituto de Alta Investigación, Universidad de Tarapacá Arica, Chile. ${ }^{2}$ Escuela de Salud Pública, Facultad de Medicina, Universidad de Chile.

${ }^{3}$ Programa de Genética Humana, ICBM, Facultad de Medicina, Universidad de Chile.

${ }^{4}$ Departamento de Psiquiatría, Escuela de

Medicina Pontificia

Universidad Católica de Chile.

${ }^{5}$ Servicio de Neurología y Psiquiatría infantil, Hospital Luis Calvo Mackenna, Facultad de Medicina, Universidad de Chile.

aTesista de Doctorado.

Recibido el 11 de agosto de 2010, aceptado el 14 de marzo de 2011

Correspondencia a: Dr. Francisco Rothhammer Teléfono: 56-2-9786449 E-mail:frthham@med. uchile.cl

\section{Risk of attention deficit/hyperactivity disorder in Aymara and Rapa-Nui school children. Association with dopaminergic system polymorphisms}

Background: Attention deficit/hyperactivity disorder (ADHD) is a common, highly heritable neurobiological disorder of childhood onset, characterized by hyperactivity, impulsiveness or inattentiveness. Aim: To search for differences in risk for ADHD and its components among Chilean native and mixed populations and to look for possible associations with dopamine receptor D4 (DRD4) and dopamine transporter 1 (DAT1) polymorphisms. Material and Methods: School teachers were requested to complete the Conners test, which uses DSM-IV criteria, to screen for ADHD risk among Aymara and Rapa-Nui students. Results: Rapa-Nui children from Easter Island had the highest risk of hyperactivity/impulsiveness. Aymara children from the Arica-Parinacota Region had lower scores. Although inattentiveness scores had lower differences between groups, overall $A D H D$ score differences among studied populations were highly significant. DRD4 and DAT1 alleles had a heterogeneous distribution. Easter islanders had more divergent frequencies, most probably as a result of separate migration routes utilized at different time periods during the colonization of America and Polynesia. Conclusions: The comparison of ADHD risk parameters between Rapa-Nui and Aymara children showed marked differences. Allele distribution of dopamine polymorphisms in Easter Island was also significantly different from northern Chile, due probably to different colonization histories. These findings suggest that higher ADHD risk scores in Easter Island children may be linked to the presence of different DRD4 alleles.

(Rev Med Chile 2011; 139: 600-605).

Key words: Attention deficit disorder with hyperactivity; Indians, South American; Polynesia; Receptors, dopamine DH.
4 1 trastorno de déficit atencional/hiperactividad (TDAH) es una condición neuropsiquiátrica de alta prevalencia en numerosas poblaciones a nivel mundial, cuya base posiblemente se encuentre en una disfunción de la neurotransmisión dopaminérgica hacia la corteza prefrontal y los ganglios basales. Si bien la participación de factores psicosociales y familiares puede ser determinante en algunos casos, la contribución genética es evidente, alcanzando de acuerdo a 
estudios previos cerca de $80 \%{ }^{1}$.Cabe mencionar, sin embargo, que este estimador se obtuvo con base en estudios de gemelos mono y dicigóticos, no habiéndose identificado hasta el momento un gen mayor involucrado. Investigaciones relacionadas con la etiología del TDAH son de especial relevancia debido a su relación con conductas que inciden significativamente en el rendimiento escolar y universitario ${ }^{2-4}$.

Entre los genes asociados al TDAH se encuentran el receptor de dopamina DRD4 y el transportador DAT1. Si bien la asociación con estos polimorfismos considerados en forma separada es leve, en varios estudios realizados en familias de Santiago de Chile pudimos establecer una asociación altamente significativa de una combinación de genotipos (heterocigotos DRD4/7y homocigotos DAT1/10) de estos dos polimorfismos con TDAH ${ }^{5-7}$. Señalamos además que según Volkow et al, $2004^{8}$, los alelos DRD4/7 y DAT1/10 podrían inducir un funcionamiento anormal en el sentido que uno (DRD4/7) sería responsable de una respuesta subóptima y el otro (DAT1/10) de una respuesta muy eficiente, provocando el efecto combinado de ambos una acentuada depleción de dopamina en la región perisináptica. Esta hipótesis fue expuesta en forma actualizada por nuestro grupo en un trabajo previo ${ }^{7}$. De acuerdo con evidencia reciente, la interacción DRD4/DAT1 parece relacionarse con un fenotipo impulsivo y alteraciones en la capacidad ejecutiva inhibitoria en adultos sanos?.

En general, habitualmente se parte del supuesto que tanto la prevalencia descrita para TDAH en poblaciones anglosajonas (3-7,5\%) como el supuesto grado de asociación con genes dopaminérgicos es representativa a nivel mundial, en circunstancias que debido a las marcadas diferencias poblacionales de frecuencia de los genotipos dopaminérgicos previamente estudiados, deberían también existir diferencias poblacionales en el riesgo de $\mathrm{TDAH}^{10,11}$. Recientemente, los resultados de dos meta-análisis que incluyeron un abultado conjunto de poblaciones a nivel internacional ratificaron la asociación entre DRD4 y TDAH y revelaron que ésta es significativa y levemente mayor en Europa $(\mathrm{OR}=1,635)$ y Sud América $(\mathrm{OR}=2,407)$ en comparación con los paises del Medio Oriente $(\mathrm{OR}=0,717)^{12,13}$.

Por otra parte, llama la atención que estudios realizados en pueblos originarios siguen siendo escasos a pesar de la importancia que el conocimiento de la prevalencia del TDAH tiene para su prevención y la realización de intervenciones psicosociales para mejorar el rendimiento escolar de los estudiantes ${ }^{14}$.

Considerando lo expuesto, nos fijamos como objetivo indagar si existe evidencia de diferencias de riesgo de TDAH entre poblaciones chilenas, en especial entre grupos étnicos de diferente origen geográfico $\mathrm{y}$, en caso positivo, si éstas estan asociadas a la presencia de los genes del sistema dopaminérgico previamente estudiadas en Santiago de Chile.

\section{Material y Método}

El riesgo de presentar TDAH se determinó en muestras poblacionales de la etnia aymara de la precordillera de la Región de Arica-Parinacota y de la etnia rapa-nui de Isla de Pascua. La información se obtuvo en los establecimientos educacionales de las áreas geográficas respectivas comprendiendo la casi totalidad de los escolares matriculados. Los resultados logrados se compararon con aquellos previamente obtenidos por nuestro grupo de trabajo para la población mixta de la ciudad de Santiago. La obtención de los estimadores de riesgo se efectuó solicitando a los maestros de escuela aplicar la prueba de Conners abreviada para profesores ${ }^{15,16}$. Esta escala para detectar conductas asociadas al TDAH, de amplio uso, consiste en que profesores familiarizados con el comportamiento de sus alumnos, aceptan completar un cuestionario de preguntas sobre el comportamiento de éstos en clase. A las respuestas se les asigna puntaje de acuerdo a los criterios diagnósticos del DSM-IV, que posteriormente permite evaluar tanto el riesgo de presentar TDAH como tambien algunos de sus componentes tales como déficit atencional (DA), hiperactividad $(\mathrm{H})$, impulsividad (I), oposicionismo desafiante (OD) o problemas emocionales (PE). Los protocolos correspondientes a diferentes fases de este proyecto de investigación fueron aprobados por el Comité de Ética de la Facultad de Medicina de la Universidad de Chile.

Como previamente hemos señalado, el objetivo central del presente trabajo guarda relación con la contrastación de dos hipótesis. Procederemos en primer lugar a verificar si existe evidencia de diferencias de riesgo de TDAH entre poblaciones chilenas, en especial entre grupos étnicos 
de diferente origen geográfico. En caso positivo, estudiaremos la posibilidad que las diferencias detectadas estén asociadas a la presencia de genes del sistema dopaminérgico previamente estudiadas en Santiago de Chile.

Para lograr nuestro objetivo, el riesgo de TDAH de estudiantes aymaras de la precordillera de la Región de Arica-Parinacota, rapa-nui de Isla de Pascua y del área norte de Santiago de Chile fue analizado estadísticamente y comparado con las frecuencias alélicas de polimorfismos genéticos del sistema dopaminérgico correspondiente a las mismas poblaciones.

El tratamiento estadístico de la información obtenida incluyó tanto un análisis cualitativo como uno cuantitativo. Se calcularon medidas de tendencia central según género para los puntajes obtenidos en las diferentes categorías en las tres poblaciones. Los criterios empleados para fijar los puntos de cortes en los puntajes obtenidos para DA, H y PE se basaron en la experiencia clínica adquirida por los profesionales a cargo del diagnóstico de riesgo para TDAH integrantes de nuestro equipo de trabajo. Con el propósito de obtener un estimador que resumiera el riesgo de TDAH, utilizamos un tercer enfoque analítico estableciendo de acuerdo a la experiencia adquirida por nuestros profesionales un punto de corte para la suma de los puntajes obtenidos para DA, $\mathrm{H}$ y $\mathrm{PE}^{5}$. Fueron clasificados como candidatos a tener TDAH los niños que alcanzaban 15 o más puntos.

\section{Resultados}

La muestra de escolares estudiada no incluía alumnos de primero básico de la población aymara y tampoco alumnos de noveno, décimo, undécimo y decimosegundo de Santiago, en consecuencia la distribución de escolares analizada se tuvo que restringir a aquella que aparece en la Tabla 1. Además, como la distribución de escolares según género (Tabla 2) no difiere significativamente, los estudiantes fueron agrupados para la realización de las pruebas de significación estadística entre los grupos estudiados. Se calcularon respectivamente para DA, $\chi^{2}=25,00 \mathrm{p}=0,0148$, para $\mathrm{H}, \chi^{2}=43,88$ $\mathrm{p}=0,0006, \mathrm{y}, \mathrm{PE}=\chi^{2}=39,4 \mathrm{p}=0,0025$. Llama la atención el bajo porcentaje de DA en los alumnos aymara y el alto puntaje de $\mathrm{H}$ en los estudiantes rapa-nui.

Se analizaron además los puntajes como información cuantitativa obteniendo promedios y desviaciones estándar (Tabla 3 ). Resulta evidente que en todas las poblaciones el promedio de puntaje de los varones exhibe valores superiores al de las niñas. Esta tendencia concuerda con los resultados observados en poblaciones anglosajonas ${ }^{17}$. Por otra parte, es importante destacar que la población de estudiantes de Santiago presenta promedios de puntaje más bajos de riesgo de $\mathrm{H}$ que los niños aymaras y rapa-nui. El DA es semejante en las tres poblaciones estudiadas y los puntajes de PE son más altos en los niños rapa-nui.

El presente análisis reveló que los estudiantes con puntajes iguales a 15 o superiores alcanzan $8,3 \%$ entre los aymaras de la Precordillera de la XV Región; 9,7\% entre los estudiantes de Santiago y $20,8 \%$ entre los estudiantes rapa-nui. Estas diferencias son altamente significativas $\left(\chi^{2}=23,38\right.$ $\mathrm{p}=0,0001)$.

El análisis de asociación ordinal muestra a través de coeficientes de correlación de Spearman de 0,690 para $\mathrm{H}$ vs $\mathrm{DA}, 0,618$ para $\mathrm{PE}$ vs $\mathrm{H}$ y 0,525 para DA vs PE $(p<0,0001$ en cada caso $)$

Tabla 1. Distribución del número de escolares incluidos en el presente estudio

\begin{tabular}{|c|c|c|c|c|c|c|c|c|}
\hline \multirow[t]{2}{*}{ Localidad } & \multicolumn{7}{|c|}{ Curso } & \multirow[t]{2}{*}{ Total } \\
\hline & 2 & 3 & 4 & 5 & 6 & 7 & 8 & \\
\hline Isla de Pascua & $\begin{array}{c}62 \\
11,61\end{array}$ & $\begin{array}{c}71 \\
13,30\end{array}$ & $\begin{array}{c}73 \\
13,67\end{array}$ & $\begin{array}{c}72 \\
13,48\end{array}$ & $\begin{array}{c}97 \\
18,16\end{array}$ & $\begin{array}{c}90 \\
16,85\end{array}$ & $\begin{array}{c}69 \\
12,92\end{array}$ & 534 \\
\hline $\begin{array}{l}\text { Precordillera } \\
\text { XV Región }\end{array}$ & $\begin{array}{c}16 \\
22,22\end{array}$ & $\begin{array}{c}13 \\
18,06\end{array}$ & $\begin{array}{c}16 \\
22,22\end{array}$ & $\begin{array}{c}0 \\
0,00\end{array}$ & $\begin{array}{c}0 \\
0,00\end{array}$ & $\begin{array}{c}14 \\
19,44\end{array}$ & $\begin{array}{c}13 \\
18,06\end{array}$ & 72 \\
\hline Santiago & $\begin{array}{c}41 \\
11,08\end{array}$ & $\begin{array}{c}35 \\
9,46\end{array}$ & $\begin{array}{c}44 \\
11,89\end{array}$ & $\begin{array}{c}65 \\
17,57\end{array}$ & $\begin{array}{c}69 \\
18,65\end{array}$ & $\begin{array}{c}63 \\
17,03\end{array}$ & $\begin{array}{c}53 \\
14,32\end{array}$ & 370 \\
\hline Total & 119 & 119 & 133 & 137 & 166 & 167 & 135 & 976 \\
\hline
\end{tabular}


que DA, H y PE están altamente correlacionados. La distribución condicional de probabilidad de DA según nivel de $\mathrm{H}$ es diferente a la distribución condicional de probabilidad de $\mathrm{H}$ según nivel de DA. En la primera, el centro de gravedad se va desplazando hacia valores mayores de $\mathrm{H}$ ilus-

Tabla 2. Distribuciones de escolares según género incluidos en el presente estudio

\begin{tabular}{|lccc|}
\hline Localidad & \multicolumn{2}{c}{ Sexo } & Total \\
\hline Isla de Pascua & F & M & \\
& 46,63 & 285 & 534 \\
Precordillera & 40 & 32 & 72 \\
XV Región & 55,56 & 44,44 & \\
Santiago & 197 & 173 & 370 \\
& 53,24 & 46,76 & \\
Total & 486 & 490 & 976 \\
\hline
\end{tabular}

trando la asociación altamente significativa que detectó el análisis de correlación de Spearman. En cambio la segunda, igualmente significativa, es menos intensa en cuanto al desplazamiento del centro de gravedad parcial, insinuando que $\mathrm{H}$ es posiblemente un mejor predictor de DA que DA lo es de $\mathrm{H}$.

Al observar la Tabla 4, que muestra las frecuencias génicas de los alelos de los sistemas genéticos dopaminérgicos, resulta evidente que las tres poblaciones presentan frecuencias diferentes. La significación estadística de las diferencias de frecuencias de los alelos fue evaluada a través del método de simulación de Monte Carlo, utilizando tablas de contingencia RxC, basadas en el algorítmo de Roff y Bentzen $(1989)^{18}$, que utiliza 10.000 replicaciones de permutaciones de alelos entre los grupos. Los resultados obtenidos para los alelos $\mathrm{DRD} 4 / 2$ y DRD4/7 fueron respectivamente $\chi^{2}=$ $136,2 \mathrm{p}<0,000$ y $\chi^{2}=35,5 \mathrm{p}<0,000$. Consideran-

Tabla 3. Promedios y desviaciones estándar de los puntajes obtenidos en la escala de Conners según localidad y género

\begin{tabular}{|llrccc|}
\hline Población & Sexo & n & Hiperactividad & Déficit atencional & Problemas emocionales \\
Isla de Pascua & Femenino & 249 & $1,76 \pm 2,34$ & $1,47 \pm 1,79$ & $0,87 \pm 1,62$ \\
Isla de Pascua & Masculino & 285 & $3,78 \pm 3,08$ & $2,61 \pm 2,11$ & $1,81 \pm 2,37$ \\
Precordillera XV Región & Femenino & 40 & $1,33 \pm 1,98$ & $1,35 \pm 1,42$ & $0,38 \pm 0,84$ \\
Precordillera XV Región & Masculino & 32 & $3,62 \pm 2,83$ & $2,41 \pm 1,78$ & $0,72 \pm 1,28$ \\
Santiago & Femenino & 197 & $1,41 \pm 2,03$ & $1,74 \pm 1,80$ & $0,73+1,48$ \\
Santiago & Masculino & 172 & $2,63 \pm 2,53$ & $2,20 \pm 1,76$ & $0,76 \pm 1,46$ \\
\hline
\end{tabular}

Tabla 4. Distribución de alelos de los sistemas genéticos dopaminérgicos DRD4 y DAT 1 en las localidades estudiadas

\begin{tabular}{|c|c|c|c|c|c|c|c|c|c|c|}
\hline Población & n & DRD4.2 & DRD4.3 & DRD4.4 & DRD4.5 & DRD4.6 & DRD4.7 & DRD4.8 & DRD4.9 & DRD4.10 \\
\hline Isla de Pascua & 64 & 0,25 & 0,11 & 0,53 & 0,02 & 0 & 0,10 & 0 & 0 & 0 \\
\hline $\begin{array}{l}\text { Precordillera } \\
\text { XV Región }\end{array}$ & 83 & 0 & 0 & 0,57 & 0,13 & 0,03 & 0,27 & 0 & 0 & 0 \\
\hline Santiago & 100 & 0,06 & 0,01 & 0,59 & 0,02 & 0,05 & 0,27 & 0,01 & 0,01 & 0 \\
\hline Población & $\mathbf{n}$ & DAT1.3 & DAT1.5 & DAT1.7 & DAT1.8 & DAT1.9 & DAT1.10 & DAT1.11 & DAT1.12 & DAT1.13 \\
\hline Isla de Pascua & 64 & 0 & 0 & 0 & 0 & 0,12 & 0,87 & 0,01 & 0 & 0 \\
\hline $\begin{array}{l}\text { Precordillera } \\
\text { XV Región }\end{array}$ & 83 & 0 & 0 & 0 & 0 & 0,05 & 0,95 & 0 & 0 & 0 \\
\hline Santiago & 100 & 0 & 0 & 0 & 0,01 & 0,23 & 0,74 & 0,035 & 0 & 0 \\
\hline
\end{tabular}


do pares de poblaciones, todas las comparaciones, tanto para DRD4 como para DAT1, resultaron estadísticamente significativas.

\section{Discusión}

Nuestros resultados indican que existen diferencias significativas entre los estimadores de DA, $\mathrm{H}$ y $\mathrm{PE}$ para las tres poblaciones chilenas estudiadas. Si analizamos los puntajes como variables cuantitativas, los promedios de puntajes de los alumnos aymara se acercan a los niños rapa-nui, siendo ambos mayores que los de los estudiantes de Santiago.

El porcentaje de alumnos aymara con puntajes superiores o iguales a 15 considerando todas las categorías (H, DA y PE) es el más bajo (8,3\%). Cabe mencionar que en un estudio previo encontramos porcentajes aun más bajos $(7,2 \%)$ en alumnos aymaras del Valle de Azapa ${ }^{19}$. Luego se ubican los estudiantes de Santiago con 9,7\% y los niños rapanui con $20,8 \%$. El empleo de un punto de corte en la distribución de puntajes totales agrupa a los niños aymara, tanto del Valle de Azapa como de la Precordillera de la XV Región con los de Santiago, teniendo los niños rapa-nui un riesgo mayor de presentar TDAH.

$\mathrm{Al}$ analizar la fuerte asociación entre $\mathrm{H}$ y DA y la tendencia descubierta de que $\mathrm{H}$ es aparentemente un mejor predictor de DA que DA de $\mathrm{H}$, resulta interesante destacar que en nuestro estudio es más difícil encontrar niños que presentan riesgo de hiperactividad/impulsividad (H/I) sin DA que viceversa y que los genes que hemos encontrado asociados al TDAH en estudios previos codifican para receptores y transportadores de dopamina que posiblemente estén asociados a $\mathrm{H} / \mathrm{I}^{4}$.

Con respecto a los genes dopaminérgicos destacan las diferencias más marcadas que separan a la muestra de Isla de Pascua de las muestras de la Precordillera de la XV Región y de Santiago. En consecuencia se podría plantear la hipótesis que los niños rapa-nui podrían tener un riesgo más alto de TDAH debido a la presencia de ciertos alelos menos frecuentes o ausentes en otras poblaciones chilenas como por ejemplo el alelo DRD4/2. En este contexto y considerando el origen polinésico de la población rapa-nui, es interesante mencionar la asociación de TDAH con el mismo alelo en poblaciones asiáticas ${ }^{20}$. Si bien estas últimas dieron origen tanto a los grupos amerindios como a las poblaciones polinésicas, el vínculo genético con éstas últimas es mucho más reciente. En efecto la colonización de América del Sur a partir de Asia se produjo hace aproximadamente 14.000 años, en circunstancias que el poblamiento de Polinesia ocurrió hace menos de 2.500 años ${ }^{21}$.

En términos más generales y concluyendo, podríamos plantear la hipótesis que la mayor prevalencia de TDAH en varones se debe en parte a la presencia de complejos de genes ligados limitados parcialmente al sexo, que determinan una mayor H/I. La frecuencia más alta de estos genes en algunas poblaciones podría estar relacionada con el mayor valor adaptativo darviniano (VAD) que les confiere la conducta que en parte determinan. Este mayor VDA podría haber sido particularmente importante en fases tempranas de nuestra historia evolutiva ${ }^{4,22}$.

Agradecimientos: Agradecemos a los profesores de los colegios de Isla de Pascua, Región de Arica-Parinacota y Región Metropolitana por su entusiasta participación en el presente estudio. Cabe destacar la desinteresada colaboración de los revisores anónimos que ayudaron a mejorar sustancialmente este trabajo, que fue financiado a través de fondos otorgados por los Proyecto Fondecyt No 1080219 y Convenio de Desempeño UTA/Mecesup 2.

\section{Referencias}

1. Faraone SV, Doyle AE. The nature and heritability of attention-deficit/hyperactivity disorder. Child Adolesc Psychiatr Clin N Am 2001; 10: 299-316.

2. Birchwood J, Daley D. Brief report: The impact of attention deficit hyperactivity disorder (ADHD) symptoms on academic performance in an adolescent community sample. J Adolesc 2010 Sep 27 [Epub ahead of print].

3. Ek U, Westerlund J, Holmberg K, Fermell E. Academic performance of adolescents with ADHD and other behavioural and learning problems-a population- based longitudinal study. Acta Paediatr 2010 [Epub ahead of print].

4. Ding YC, Chi HC, Grady DL, Morishima A, Kidd JR, Kidd KK. From the Cover: Evidence of positive selection acting at the human dopamine receptor D4 gene locus. Proc Nat Acad Sci USA 2002; 99: 309-14.

5. Carrasco X, Rothhammer P, Moraga M, Henríquez H, Aboitiz F, Rothhammer F. [Presence of DRD4/7R and 
DAT1/10R allele in Chilean family members with attention deficit hyperactivity disorder]. Rev Med Chile 2004; 132: 1047-52.

6. Carrasco X, Rothhammer P, Moraga M, Henríquez H, Chakraborty R, Aboitiz F, Rothhammer F. Genotypic interaction between DRD4 and DAT1 loci is a high risk factor for attention-deficit/hyperactivity disorder in Chilean families. Am J Med Genet B Neuropsychiatr Genet 2006; 141: 51-4.

7. Henríquez H, Henríquez M, Carrasco X, Rothhammer P, Llop E, Aboitiz F, et al. Combinación de Genotipos DRD4 y DAT1 Constituye Importante Factor de Riesgo en Miembros de Familias de Santiago de Chile con Déficit Atencional. Rev Med Chile 2008; 136: 719-24.

8. Volkow ND, Wang GJ, Fowler JS, Telang F, Maynard L, Logan J, et al. Methylphenidate enhances the saliency of a mathematical task by increasing dopamine in the human brain. Am J Psychiatry 2004; 161: 1173-80.

9. Congdon E, Lesch KP, Canli T. Analysis of DRD4 and DAT polymorphisms and behavioural inhibition in healthy adults: Implications for impulsivity. Am J Med Genet B Neuropsychiatr Genet 2008; 147: 27-32.

10. Vieyra G, Moraga M, Henríquez H, Aboitiz F, Rothhammer F. [Distribution of DRD4 and DAT1 alleles from dopaminergic system in a mixed Chilean population]. Rev Med Chile 2003; 131: 135-43.

11. Bobb AJ, Castellanos FX, Addington AM, Rapoport JL. Molecular genetic studies of ADHD: 1991 to 2004. Am J Med Genet Part B (Neuro Psychiatr Genet) 2005; 132: 109-25.

12. Faraone SV, Mick E. Molecular genetics of attention deficit hyperactivity disorder. Psychiatr Clin North Am 2010; 33: 159-80

13. Nikolaidis A, Gray JR. ADHD and the DRD4 exon 111 7-repaet polymorphism: an international meta-analysis. Soc Cogn Affect Neurosci 2010; 5 (2-3): 188-93.

14. Tresco KE, Lefler EK, Power TJ. Psychosocial interventions to improve the school performance of students with attention-deficit/hyperactivity disorder. Mind Brain 2010; 1: 69-74.

15. Conners CK. Conners Rating Scales-Revised. Technical Manual. Toronto: Multi-Health Systems. 1997.

16. Wichs-Nelson R, Israel A. Psicopatología del Niño y del Adolescente. 3a Ed. Prentice, Hall, Madrid, 1999.

17. Taylor E. Clinical foundations of hyperactivity research. Behav Brain Res 1998; 94: 11-24.

18. Roff D, Bentzen P. The statistical analysis of mitochondrial DNA polymorphism: $c 2$ and the problem of small samples. Mol Biol Evol 1989; 6: 539-45.

19. Rothhammer P, Carrasco X, Henríquez H, Andrade C, Valenzuela M, Aboitiz F, et al. Bajo riesgo de déficit atencional/Hiperactividad en niños Aymarás. Implicancias genéticas, antropológicas y culturales. Chungará Rev Antropol Chilena 2005; 37: 145-9.

20. Leung PW, Lee CC, Hung SF, Ho TP, Tang CP, Kwong SL, et al. Dopamine receptor D4 (DRD4) gene in Han Chinese children with attention-deficit/hyperactivity disorder (ADHD): increased prevalence of the 2-repeat allele. Am J Med Genet B Neuropsychiatr Genet 2005 133: 54-6.

21. Rothhammer F, Dillehay TD. The Late Pleistocene colonization of South America: An interdisciplinary perspective . Ann.Hum.Genet 2009; 73: 540-9.

22. Ben Zion IZ, Tessler R, Cohen L, Lerer E, Raz Y, BachnerMelman R, et al. Polymorphisms in the dopamine D4 receptor gene (DRD4) contribute to individual differences in human sexual behavior: desire, arousal and sexual function. Mol Psychiatry 2006; 11: 782-6. 